\title{
Les Lumières radicales. La Philosophie, Spinoza et la naissance de la modernité (1650-1750)
}

\section{Marc Belissa}

\section{(2) OpenEdition \\ 1 Journals}

Édition électronique

URL : https://journals.openedition.org/ahrf/7333

DOI : 10.4000/ahrf.7333

ISSN : 1952-403X

\section{Éditeur :}

Armand Colin, Société des études robespierristes

\section{Édition imprimée}

Date de publication : 1 septembre 2006

Pagination : 204-208

ISSN : 0003-4436

\section{Référence électronique}

Marc Belissa, « Les Lumières radicales. La Philosophie, Spinoza et la naissance de la modernité

(1650-1750) ", Annales historiques de la Révolution française [En ligne], 345 I juillet-septembre 2006, mis en ligne le 08 juillet 2008, consulté le 01 juillet 2021. URL : http://journals.openedition.org/ahrf/7333 ; DOI : https://doi.org/10.4000/ahrf.7333

Ce document a été généré automatiquement le 1 juillet 2021.

Tous droits réservés 


\title{
Les Lumières radicales. La Philosophie, Spinoza et la naissance de la modernité (1650-1750)
}

\author{
Marc Belissa
}

\section{RÉFÉRENCE}

Jonathan Israel, Les Lumières radicales. La Philosophie, Spinoza et la naissance de la modernité (1650-1750), Paris, Éditions Amsterdam, 2005, 935 p., ISBN 2-915547-12-2, $37 €$.

1 Cet ouvrage de Jonathan Israel est la traduction de Radical Enlightenment, Philosophy and the Making of Modernity publié en 2001 et déjà devenu un classique de l'historiographie dans le monde anglo-saxon. Le projet de l'auteur peut être décrit comme un triple décentrage qui entend aboutir à une révision complète de la compréhension du phénomène des Lumières.

2 Décentrage géographique tout d'abord: refusant les francocentrisme et anglocentrisme des interprétations classiques, ainsi que l'idée de Lumières " nationales ", Israel insiste sur le caractère européen et unifié du débat philosophique dans la période qu'il considère comme celle de l'émergence de la "modernité ». Spécialiste des ProvincesUnies, l'auteur met l'accent sur la pensée hollandaise, mais il intègre également dans sa démonstration la péninsule ibérique, l'aire baltique, l'Allemagne et l'Italie. Analysant des textes et des auteurs peu ou très peu connus, Jonathan Israel dresse un panorama érudit et virtuose des courants philosophiques, des débats religieux et politiques qui opposent les théologiens, les philosophes d'Europe occidentale.

Décentrage chronologique ensuite. L'auteur remonte bien au-delà de la période de la «crise de conscience européenne » des années 1680-1690. Pour Jonathan Israel, c'est la période 1650-1750 qui forme le cœur des Lumières, intégrant donc dans un même ensemble l'âge d'or du rationalisme classique (Descartes, Spinoza, Leibniz, Newton...) et celui des Lumières proprement dites (Voltaire, Wolff, Rousseau, Diderot...). De manière 
tranchée, Jonathan Israel affirme que les développements des Lumières après 1750 ne sont "qu'une consolidation, une vulgarisation et un commentaire des concepts révolutionnaires introduits précédemment » (p. 31).

Décentrage intellectuel enfin puisque Israel entend montrer l'influence déterminante des «marges des Lumières ", à savoir la pensée panthéiste de Spinoza et des spinozistes sur le "centre ", c'est-à-dire le quatuor Newton, Locke, Voltaire et Montesquieu. En effet, Israel affirme tout au long de son ouvrage que la pensée de Spinoza a provoqué une onde de choc européenne et que toute la philosophie ultérieure a dû se confronter à cette pensée qualifiée de «radicale ». Ainsi, il distingue quatre courants à l'origine des Lumières : un courant newtonien, un courant néo-cartésien, un courant leibnizien et un courant spinoziste ou radical, mais c'est ce dernier qui aurait joué un rôle moteur dans la dynamique qui a présidé au renouveau intellectuel de la période, les autres courants réagissant au spinozisme.

5 Ces courants se recomposent en fonction de leur rapport à l'ordre ancien : l'auteur considère ainsi trois pôles, un pôle " radical-spinoziste ", un pôle " modéré » cherchant le compromis entre les Lumières et la société d'Ancien Régime et enfin un pôle "conservateur", mais influencé par les Lumières. Les Lumières sont donc ainsi traversées par une lutte intellectuelle et politique entre ces trois pôles, le pôle «modéré » s'alliant au pôle « conservateur » contre le pôle radical-spinoziste dont les coups de boutoir ont ébranlé l'édifice intellectuel de l'Ancien Régime et ont préparé l'irruption de la « modernité » et de la Révolution.

6 Ce qui caractérise la «radicalité» des spinozistes, c'est leur refus absolu de la révélation et le fait qu'ils s'appuient exclusivement sur la raison pour analyser les faits naturels, politiques et sociaux. Le dualisme cartésien de la matière et de l'esprit est rejeté. L'univers ne forme qu'une seule substance dont la pensée est une des manifestations. Cette radicalité implique un rejet du compromis avec les autorités religieuses, intellectuelles, politiques ou sociales. Les Lumières « radicales" sont donc antireligieuses, antimonarchiques et anti-aristocratiques. Contrairement à l'interprétation «darntonienne » des Lumières, Israel considère qu'un lien unit la radicalité philosophique des spinozistes et le radicalisme politique révolutionnaire. Le pôle « modéré » entend au contraire s'accommoder de l'Ancien Régime tout en tentant de le "réformer ", ce sont les Lumières d'État, mais aussi les diverses formes de déisme qui admettent les magistères séparés de la révélation et de la raison. D’un côté donc Newton, Locke, Voltaire ou Montesquieu, de l'autre Spinoza, Boulainvilliers, Dumarsais, Diderot ou le baron d'Holbach.

7 L'ouvrage est organisé en cinq parties qui ne se suivent pas de manière chronologique ni strictement thématique: la première se présente comme une vision d'ensemble. L'auteur y étudie tout d'abord la diffusion du cartésianisme et les réactions des pouvoirs face à la nouvelle philosophie, les formes de la circulation et de la censure des textes radicaux, et enfin il aborde la question sous l'angle du genre. Israel voit ces évolutions comme un élément d'un contexte intellectuel marqué par les événements du milieu du XVII siècle : Fronde, révolte de Naples et Révolution anglaise. À partir de la deuxième partie, l'auteur revient à une construction semi-chronologique en partant de son «héros»: Spinoza. Israel décrit ensuite la diffusion de sa pensée dans les Provinces-Unies. La troisième partie élargit cette recherche à l'ensemble de l'Europe. La quatrième partie est consacrée à la réponse européenne au défi spinoziste, Israel y développe l'idée que les philosophies de Leibniz, de Locke ou de Wolff sont des réponses 
«modérées" au spinozisme. La cinquième partie est consacrée à "la progression clandestine des Lumières radicales 1680-1750" dans toute l'Europe et le chapitre conclusif s'ouvre sur Rousseau et sa postérité révolutionnaire.

On aura compris que la figure centrale de l'ouvrage est celle de Spinoza et incontestablement l'un des mérites principaux de l'ouvrage est de restituer à sa philosophie sa dimension européenne, mais aussi sociale et politique. L'auteur insiste sur le fait que Spinoza n'est pas un penseur isolé, mais qu'il est inséré dans un débat hollandais d'abord, européen ensuite, et qu'il donne naissance à un courant philosophique et politique délimité. Israel cherche - et trouve - l'influence de Spinoza dans toute l'Europe, chez les Commonwealthmen, en Italie avec Vico, en Allemagne dans l'Aufklärung radicale, en France chez Diderot ou d'Holbach. L'autre grand mérite de cet ouvrage est la vision réellement européenne qu'il impose (mais on pourra regretter l'absence totale de Genève dont l'importance philosophique n'est pas proportionnelle à la taille de la cité), ainsi que le décentrage chronologique convaincant proposé comme cadre indispensable pour la compréhension de l'émergence des Lumières. Les Provinces-Unies de la deuxième moitié du XVII ${ }^{e}$ siècle regagnent ainsi une place déterminante dans l'histoire des idées en Europe.

9 Si l'importance d'un ouvrage se mesure au débat et aux critiques qu'il suscite, Les Lumières radicales sont de toute évidence un livre capital, car les critiques, parfois acerbes, n'ont pas manqué. Elles ont porté à la fois sur les résultats de la recherche, mais aussi sur sa démarche et sa méthode.

Beaucoup d'historiens anglo-saxons ont d'abord remarqué que la lecture des textes philosophiques par Israel faisait l'impasse sur le renouvellement des méthodes de traitement des sources culturelles et intellectuelles. En effet, l'auteur ne cache pas sa volonté de faire une "histoire des idées ", à l'ancienne pourrait-on dire, une histoire philosophique et intellectuelle appuyée sur une lecture très classique des textes. De fait, on a souvent l'impression de lire une histoire des idées désincarnée, décontextualisée où les influences des penseurs les uns sur les autres sont considérées de manière déterministe. Pourtant si telle idée développée par Spinoza se retrouve chez tel ou tel auteur, faut-il y voir nécessairement du " spinozisme »? L'auteur a bien souvent cette tendance à le voir chez tous et partout. Que la pensée de Spinoza ait été une des «traditions » auxquelles les intellectuels des Lumières se soient confrontés est une certitude, mais à l'image de ce qu'est celle de Hobbes, par exemple, elle sert surtout de repoussoir et d'élément dans un processus d'élaboration philosophique. Spinoza et Hobbes sont en effet les "épouvantails » des Lumières contre lesquels tout le monde vitupère, mais que tout le monde utilise. "Spinoza " et "spinozistes" sont ainsi souvent des topoi ou des caractérisations vagues. Déclarer que tel écrit est «spinoziste » relève donc souvent de l'insulte au XVIII siècle et non de l'analyse. La pensée de Spinoza est incontestablement un des éléments constitutifs du débat des Lumières, mais elle n'est que cela. Les philosophes du XVIII e siècle dialoguent avec Spinoza, mais aussi avec Grotius, Pufendorf, Leibniz et Locke.

11 Les spécialistes des Lumières «tardives » et de la Révolution s'étonneront par ailleurs de l'affirmation liminaire de l'auteur selon laquelle rien de neuf n'émerge des débats philosophiques après 1750. Certes, on sait depuis Ernst Cassirer et d'autres à quel point les Lumières discutent et utilisent les concepts " inventés " précédemment, mais ce travail de confrontation et de recomposition n'est pas une compilation stérile. L'œuvre de Rousseau, pour ne citer qu'elle, en est la preuve. Et que dire de la philosophie en 
action de la Révolution française? N'innove-t-elle en rien? Comment écrire que « Morellet, Mably, Mirabeau, d'Holbach, Naigeon, Maréchal, Saint-Just, Babeuf et autres utopistes, proto-socialistes ou matérialistes athées" ne sont que des «penseurs secondaires », et "parfois même dénués de toute valeur propre »? L'historien doit-il ainsi distribuer bons et mauvais points aux « bons philosophes » et aux « imitateurs »? Une autre des critiques les plus conséquentes que l'on peut faire à l'ouvrage est la dichotomie établie entre Lumières « radicales » et «modérées » et surtout la tendance permanente à la réification de la pluralité des Lumières en courants délimités. Sans aller jusqu'à considérer ces courants comme des "fractions", on a réellement l'impression que pour Israel les frontières entre ces courants sont tracées et qu'ils «agissent» en mouvements "pour soi » (au sens hégéliano-marxiste du terme). L'utilisation des termes «radical » et "modéré » est d'ailleurs en soi un problème de taille. Il y a là d'abord un effet de traduction qui n'est pas sans conséquence. En effet, le terme anglais radical n'est pas absolument identique au français « radical» qui possède une connotation presque exclusivement politique, ce qui n'est pas le cas du terme anglais.

13 Ce qui semble constituer la radicalité aux yeux de Jonathan Israel est en fait surtout le refus de la religion révélée, du déisme et de la transcendance du social et du politique. Or l'assimilation du refus de la religion révélée à un radicalisme social et politique est très loin d'être évidente. L'athéisme ou le panthéisme spinoziste sont-ils nécessairement égalitaires et démocratiques dans la période des Lumières? Les spécialistes de la Révolution française savent bien que la radicalité égalitaire et politique d'un abbé Grégoire s'accommode fort bien d'un christianisme anticlérical épuré, à l'inverse les athées déchristianisateurs sont-ils forcément les révolutionnaires les plus égalitaires? On peut en douter. Les contre-exemples peuvent être multipliés : Boulainvilliers, présenté comme un républicain radical n'est pas démocrate (ce que Israel concède volontiers d'ailleurs), d'Holbach, athée radical est très éloigné de l'égalitarisme et encore plus de l'antimonarchisme. La pensée athée peut très bien s'accommoder d'une conception aristocratique de la société qui considère que la crédulité des masses est un fait de nature. Enfin a contrario, l'égalitarisme et le républicanisme de Rousseau ne l'empêchent pas de développer une conception de la religion tout à fait opposée à celle du cercle holbachique, cela fait-il pour autant de Rousseau un «modéré »?

Remarquons enfin, que l'un des points communs aux théoriciens regroupés par Jonathan Israel sous le chapeau du « radicalisme » est qu'ils se trouvent presque tous en dehors du courant jusnaturaliste. Celui-ci implique en effet, une forme de transcendance du droit qui s'appuie à l'époque, soit sur une conception de la nature humaine comme émanation du divin, soit sur une conception morale de l'état de nature. «L'amoralisme » philosophique est-il dans ce cas plus « radical » que l'école du droit naturel? Une des affirmations les plus discutables de l'ouvrage est d'ailleurs la caractérisation de la philosophie de Locke comme une réaction " modérée " au radicalisme spinozien. Visiblement Israel considère que le droit naturel lockéen ne peut bénéficier de l'étiquette "radicale", niant de fait tout l'apport potentiellement révolutionnaire d'une pensée fondée sur l'idée d'une morale naturelle transcendante justifiant le droit à l'insurrection.

Enfin, le recentrage géographique sur les Provinces-Unies est certes salutaire, mais il amène fréquemment Israel à tordre le bâton dans l'autre sens et à minorer l'originalité 
ou l'importance des penseurs anglais (les Diggers du XVII ${ }^{\mathrm{e}}$ siècle, Toland, Collins) et français (Fontenelle). Ainsi, l'absence volontaire de références au "Moment machiavélien » de Pocock ou à la tradition du républicanisme classique et civique est remarquable. Il s'en est expliqué dans un article publié en 2004 dans le European Journal of Political Theory. Pour Israel, cette tradition ne constitue pas la source principale du républicanisme moderne occidental. En revanche, il voit chez Mably, Diderot, ou dans une certaine mesure Rousseau, un héritage de la pensée radicale et républicaine hollandaise. Selon lui, le républicanisme jacobin doit considérablement plus à la tradition "radicale» franco-hollandaise qu'au républicanisme "civique» angloaméricain. Pour ma part, il me semble qu'opposer ces deux sources en une querelle de « filiations » est un exercice assez vain. L'élaboration des républicanismes « modernes » ne peut se comprendre sans intégrer l'ensemble des traditions antérieures et surtout sans penser la manière dont elles se combinent en fonction de leur utilisation par les acteurs politiques dans des contextes particuliers.

On l'aura compris, l'ouvrage de Jonathan Israel est d'ores et déjà un classique incontournable pour qui s'intéresse aux Lumières, un livre d'une grande richesse érudite, mais il s'agit avant tout d'un livre qui suscite le débat et ouvre des perspectives plus qu'il ne constitue une synthèse satisfaisante sur l'origine des Lumières. Le triple recentrage proposé par Jonathan Israel est incontestablement stimulant, mais il reste à étudier plus finement les manières dont le spinozisme se combine avec les autres traditions philosophiques et comment s'opère sa contextualisation politique, discursive et culturelle. 